\title{
Reanimación cardiopulmonar en prono: una mirada histórica
}

\author{
Historical view: prone cardiopulmonary resuscitation
}

Patricio Ramiro Aravena ${ }^{1}$

\begin{abstract}
The SARS-CoV-2 pandemic that we are currently experiencing has produced new clinical scenarios, within these, prone ventilation is one of the most frequent, especially in critically ill patients, exposing us to having to perform CPR on a patient who is in a prone position. The first suggestion of a prone CPR technique was made by McNeil in 1989. To date, there have been several cases of prone CPR described, most using the technique described by McNeil or small variations of these, achieving success in resuscitation. The technique consists of positioning oneself over the patient who is in a prone position, sitting on his buttocks and resting our hands on the back of the chest and then compressing the chest using a forward swinging motion at an average speed of 40 compressions per minute, with the aim of allowing passive re-expansion of the chest. At the same time, to maintain a patent airway, either victim's arms should be positioned below their forehead so that the bridge of the nose rests on the flexion crease of the elbow, with the nose pointing directly downward. For the defibrillation in prone, two possible alternative positions of the patches/ paddles have been described that have been used successfully: position one of the patches under the patient in prone, in relation to the area of greatest ventricular mass, and the other in his back, specifically on the right shoulder blade. The second one used two patches positioned on the patient's back, one in the lower left region just posterior to the mid-axillary line and the other just below the right shoulder blade. It has been shown that the compressions performed in the prone would be as or more effective than those performed in the supine position with the standard technique, the prone technique also presenting the benefit of producing passive ventilation simultaneously with the same compression maneuver. The situation we are living in deserves to take all the tools we have, so this technique presents a viable alternative to perform in clinical practice, however, more studies are needed in this regard to establish if
\end{abstract}

Key words:

Prone,

$C P R$,

COVID-19

Médico cirujano, Licenciado en Medicina Universidad Mayor.

Fecha de recepción: 03 de agosto de 2020

Fecha de aceptación: 24 de agosto de 2020

ORCID

https://orcid.org/0000-0001-9336-568X

Correspondencia:

Patricio Ramiro Aravena

patricioramiro2@gmail.com 
there is a real benefit from this technique regarding the classical technique. Expert recommendations for CPR have emerged in the context of the COVID-19 patient in which there is consensus that it is reasonable to initiate resuscitation in the prone position in COVID-19 patients who are intubated and ventilated in this same position.

\section{RESUMEN}

La pandemia de SARS-CoV-2 que vivimos actualmente ha producido nuevos escenarios clínicos, dentro de estos la ventilación en prono es uno de los más frecuentes, sobre todo en pacientes críticos, exponiéndonos a tener que realizar una RCP a un paciente que se encuentra en prono. La primera sugerencia de una técnica de RCP en prono fue realizada por McNeil en 1989. A la fecha existen diversos casos descritos de RCP en prono, la mayoría utilizando la técnica descrita por McNeil o pequeñas variaciones de estas, logrando éxito en la reanimación. La técnica consiste en posicionarse sobre el paciente que se encuentra en prono, sentándose el reanimador sobre las nalgas de este y apoyando las manos sobre la parte posterior del tórax para luego comprimir el tórax mediante un movimiento de balanceo hacia adelante a una velocidad promedio de 40 compresiones por minuto, con el objetivo de permitir la reexpansión pasiva del tórax. A la vez, para mantener la vía aérea permeable se debe posicionar cualquiera de los brazos de la víctima por debajo de su frente de manera que el puente de la nariz descansara sobre el pliegue de flexión del codo con la nariz apuntando directamente hacia abajo. Para la desfibrilación en prono se han descrito dos posibles posiciones alternativas de los parches/ paletas que han sido utilizadas de forma exitosa: posicionar uno de los parches bajo el paciente en prono, en relación a la zona de mayor masa ventricular y el otro en la espalda de este, específicamente sobre la escápula derecha; utilizar dos parches posicionados en la espalda del paciente, uno en la región izquierda baja justo posterior a la línea axilar media y el otro justo bajo la escápula derecha. Se ha evidenciado que las compresiones realizadas en prono serían tanto o más efectivas que las realizadas en supino con la técnica estándar, presentando la técnica en prono también el beneficio de producir una ventilación pasiva de forma simultánea con la misma maniobra de compresión. La situación que vivimos amerita tomar todas las herramientas con las que contamos, por lo que esta técnica presenta una alternativa viable de realizar en la práctica clínica, sin embargo, hacen falta más estudios al respecto para poder establecer si existe un real beneficio de esta técnica respecto a la técnica clásica. Han surgido recomendaciones de expertos para la RCP en el contexto del paciente COVID-19 en las cuales existe consenso respecto a que es razonable iniciar la reanimación en posición prona en pacientes COVID-19 que se encuentran intubados y siendo ventilados en esta misma posición.

\section{Palabras clave:}

PCR en prono, COVID-19

\section{Introducción}

Linats a reanimación cardiopulmonar (RCP) es una de las competencias más necesarias en la práctica médica, tanto en la labor del anestesiólogo, el intensivista y de todo profesional médico, especialista o no. La situación actual está poniendo a prueba constantemente estas habilidades $y$, además, a presentado nuevos desafíos a los profesionales que se han visto enfrentados al manejo de pacientes COVID-19 teniendo que adquirir conocimientos de una forma acelerada para poder hacer frente al rápido avance de 
la pandemia. Dado la recomendación actual e importante beneficio que ha demostrado la posición prono en los pacientes COVID-19, su amplia indicación y la siempre existente posibilidad de tener que enfrentarse a un paro cardiorrespiratorio en estos pacientes, es que parece de suma importancia el revisar los conocimientos con los que contamos hasta el momento sobre la reanimación cardiopulmonar en prono para, de esta manera, estar mejor preparados para poder abordar esta situación de la más efectiva manera posible con el objetivo de poder lograr una RCP exitosa, sobre todo cuando supinar al paciente no resulta expedito o incluso no es factible teniendo en cuenta la alta demanda asistencial actual. Para ello se ha revisado la información con la que contamos hasta ahora, intentado seleccionar lo más relevante y que pueda ser de mayor utilidad para su aplicación actual.

\section{La RCP en prono}

El primer reporte de la realización de una RCP en prono de manera exitosa data del año 1992 e incluye 2 casos, ambos ocurridos durante intervenciones neuroquirúrgicas, en los que se describe una técnica de compresión precordial reversa que consistía en posicionar la mano izquierda empuñada bajo el esternón del paciente y a la vez comprimir a nivel de la columna dorsal media, con la mano derecha del mismo reanimador en el primer caso y por el cirujano en el segundo, lográndose una reanimación exitosa en ambos casos[1].

Posteriormente, en el año 1994, se reportó el caso de un paciente que presentó un paro cardiorrespiratorio durante una cirugía de columna, realizándose una RCP exitosa en prono con una técnica similar a la mencionada previamente, con la única diferencia de que en este caso se realizaron compresiones con ambas manos sobre las escápulas del paciente sin necesidad de usar una mano como soporte, ya que este mismo se encontraba sobre una superficie rígida[2].

Luego de que en 1993 se reportara otro caso similar, también durante cirugía neurológica, ya en 1996 se reporta el primer caso de un paro cardiorrespiratorio en un paciente que se encontraba siendo ventilado en prono debido a un síndrome de distrés respiratorio Agudo (SDRA) secundario a una neumonía adquirida en la comunidad; La técnica de reanimación en prono descrita en este caso consistió en que un primer reanimador apoyaba su mano extendida bajo el esternón del paciente mientras que un segundo reanimador realizaba las compresiones con ambas manos de forma similar a la técnica en supino, solo que sobre el ni- vel medio de la columna dorsal del paciente, logrando ritmo sinusal y el retorno de la circulación espontánea luego de 5 min de compresiones[3].

Estas técnicas derivan y/o son muy similares a la técnica descrita por McNeil en 1989. La técnica de McNeil propone diversos beneficios a favor de la reanimación en prono, en comparación a la técnica clásica en supino, en un esfuerzo por generar un cambio en las recomendaciones y técnicas clásicas de RCP, argumentando una muy baja efectividad demostrable de estas mismas[4]-[6].

El trabajo de McNeil pasó desapercibido hasta el artículo publicado por Stewart en el año 2002 el cual tomaba como base el método de McNeil y proponía nuevamente un cambio en las técnicas de RCP apoyándose en los reportes de casos disponibles hasta esa fecha, planteando la interrogante de si tal vez una técnica de RCP en prono pudiese ser más efectiva que las actuales técnicas en supino y llamando a que se realice mayor investigación[6].

Posteriormente, McNeil hace referencia a su trabajo previo y al de Stewart en una carta al editor donde señala que las técnicas de BLS no son del todo adecuadas, sobre todo al basar el diagnóstico de paro cardiorrespiratorio en la palpación del pulso carotideo, ya que, según lo expresado por el autor, este en muchas ocasiones sería difícil de percibir, sobre todo en pacientes hipotensos, lo que llevaría a una alta incidencia de diagnósticos de paro cardiorrespiratorio que en realidad serían errados[5].

Dentro de los posibles beneficios de la técnica previamente mencionada, descritos por Stewart y McNeil, se encontraban los siguientes[4],[6]:

- Aprendizaje más simple y mejor retención.

- Mantenimiento de la permeabilidad de la vía aérea sin requerir una constante atención a esta.

- Minimización del riesgo de aspiración de contenido gástrico.

- La ausencia de necesidad de tener que detener las compresiones para realizar la ventilación ya que la técnica proporcionaría soporte ventilatorio simultáneo a las compresiones.

- Eliminación de la necesidad de ventilación boca a boca.

- Disminución de la probabilidad de lesiones a la víctima, incluso en caso de un diagnóstico errado de paro cardiorrespiratorio.

Pese a que no ha existido mucho avance al respecto, la situación actual nos plantea un escenario en el que muchas veces tendremos a un paciente en prono sin una posibilidad de supinarlo de forma expedita y más importante aún, sin un conocimiento objetivo de 
si el hacerlo y realizar una reanimación con la técnica clásica signifique un real beneficio para el paciente en comparación a si se realizara la RCP en prono.

\section{Compresiones: ¿Dónde realizarlas?}

Existen 2 teorías para explicar cómo se produce el flujo sanguíneo durante la RCP[7]:

1) Teoría de la "bomba cardiaca": esta teoría postula que el flujo sanguíneo se produciría por compresión directa del corazón entre el esternón y la columna vertebral[7].

2) Teoría de la "bomba torácica" : esta teoría postula que el flujo sanguíneo es producto de un aumento de la presión intratorácica el cual excedería la presión vascular extratorácica y, además, que el flujo estaría limitado a una dirección venoarterial por la acción de las válvulas venosas que actuarían previniendo un flujo retrógrado[7].

La técnica descrita originalmente por McNeil hacía énfasis en el modelo de la "bomba torácica" como principal fundamento para privilegiar una técnica de $\mathrm{RCP}$ en prono ya que esta misma no solo permite la compresión de una mayor área del tórax, sino que también proporcionaría el importante beneficio de lograr una ventilación pasiva al realizarla con la técnica descrita, por una salida de aire producida al comprimir el tórax de forma similar a como ocurre con la maniobra de Heimlich, con un posterior ingreso pasivo de aire[4],[6].

La técnica descrita por McNeil proponía (Figura 1):

- Realizar las compresiones montándose sobre el paciente que se encuentra en prono sentándose el reanimador sobre las nalgas de este y apoyando las manos sobre la parte posterior del tórax[6].

- Comprimir el tórax mediante un movimiento de balanceo hacia adelante a una velocidad promedio de 40 compresiones por minuto, con el objetivo de permitir la reexpansión pasiva del tórax[6].

Existen diversos estudios que apoyan la teoría de la "bomba cardiaca" como principal mecanismo de flujo sanguíneo durante la RCP sosteniendo así que el gasto cardiaco es generado principalmente a través de compresión directa del corazón[8]. Teniendo esto en cuenta, el sitio anatómico donde se realizan las compresiones externas del tórax debiese ser aquel donde se logre comprimir el ventrículo izquierdo lo máximo posible para así maximizar el volumen de sangre eyectado; a la vez también debe evitarse una posible obstrucción del tracto de salida del ventrículo

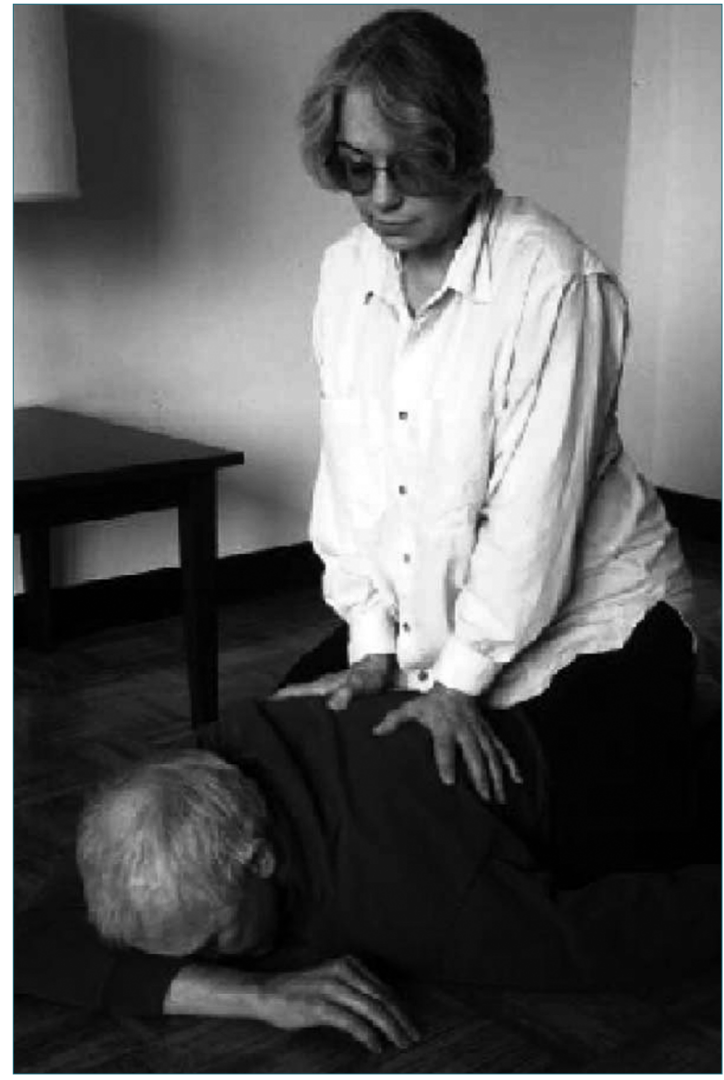

Figura 1. Reanimación de un paciente en prono.

izquierdo por las compresiones realizadas[8]. En un estudio realizado por Min-Ji Kwon et al., en el cual se analizaron tomografías computadas (TC) de 100 pacientes realizadas en prono con el objetivo de poder encontrar el sitio y referencia anatómica más adecuada para maximizar la compresión ventricular izquierda al realizar RCP en prono, se encontró que la mayor área de sección transversal del ventrículo izquierdo en la posición prono se encontraba 1 segmento vertebral por debajo del nivel del ángulo inferior de la escápula en $45 \%$ de los pacientes y entre 0 y 2 segmentos en el 95\% de los pacientes, siendo esto a nivel de T8-T9 en $79 \%$ de los pacientes estudiados (Figura 2). En 31\% de los pacientes el tracto de salida del ventrículo izquierdo se encontraba a nivel del ángulo inferior de la escápula[8]. Derivado de estos datos se evidenció que cuando el paciente está posicionado en prono la mayor área de sección transversal del ventrículo izquierdo se encuentra entre 0 a 2 segmentos vertebrales por debajo del nivel del ángulo inferior de la escápula en $86 \%$ de los pacientes[8], por lo que, podría usarse 


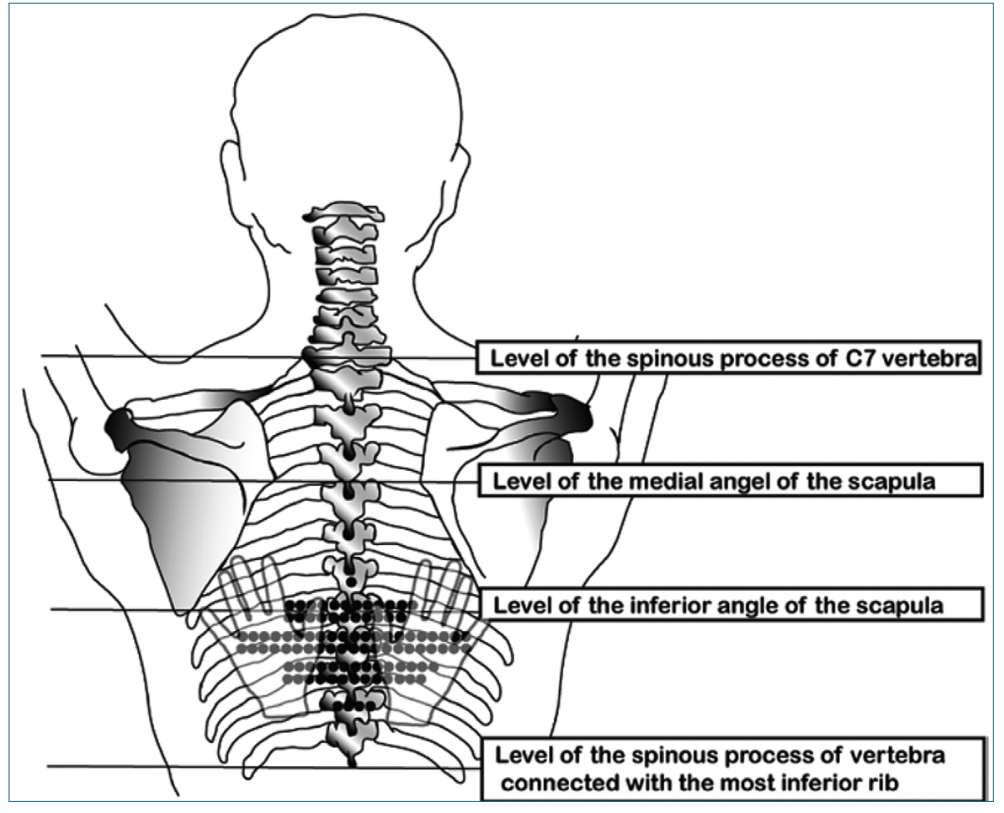

Figura 2. Nivel donde debieran realizarse las contracciones durante un paro cardiorrespiratorio. la línea imaginaria que une los ángulos inferiores de ambas escápulas como un punto de referencia anatómico que aseguraría una mayor probabilidad de realizar una compresión óptima y a la vez sería fácilmente identificable (Figura 2).

Respecto a la efectividad de las compresiones existe un estudio, realizado por Jeng Wei et al., en pacientes fallecidos en UPC el cual compara las mediciones de presión arterial, registradas mediante línea arterial, al realizar compresiones con la técnica estándar de RCP con las de las compresiones realizadas en prono sobre la columna torácica con fuerza similar a la técnica estándar, resultando las compresiones en prono en niveles de presión arterial mayores que los alcanzados con la técnica estándar[9].

\section{Vía aérea y ventilación en prono}

La pronación precoz de los pacientes asociada a ventilación con cánula nasal de alto flujo (CNAF) ha mostrado ser una alternativa razonable en el contexto actual, sobre todo ante el déficit de camas de UPC, ventiladores mecánicos adecuados y personal entrenado para usarlos. Pese al posible beneficio, siempre existe la preocupación sobre el acceso a la vía aérea en estos pacientes, sobre todo en caso de un rápido empeoramiento de la condición clínica que puede culminar en la necesidad de administrar RCP a un paciente que se encuentra en prono y sin un dispositivo avanzado que asegure la vía aérea. A modo de enfrentar estos posibles escenarios, teóricamente, parecería factible utilizar la técnica descrita por McNeil ya que permitiría la administración de RCP por un solo reanimador sin necesidad de supinar al paciente $y$, además, logrando una permeabilidad de la vía aérea y ventilación pasiva sin frenar las compresiones y sin perder tiempo valioso en supinar al paciente e instrumentalizar la vía aérea.

Para mantener la permeabilidad de la vía aérea la técnica sugería:

- Posicionar cualquiera de los brazos de la víctima por debajo de su frente de manera que el puente de la nariz descansara sobre el pliegue de flexión del codo, con la nariz apuntando directamente hacia abajo (Figura 3)[6].

- Esta posición permitiría mantener la extensión del cuello, a la vez que la mandíbula se desplaza hacia abajo y adelante, evitando una obstrucción de la vía aérea secundaria al retroceso mandibular y/o flexión pasiva del cuello como tiende a ocurrir al posicionar al paciente tanto en supino como en prono sin utilizar la técnica descrita[6],[10].

Respecto a la efectividad en la ventilación, el estudio realizado por Wei et al. previamente mencionado también analizaba la efectividad de las compresiones en prono como método de ventilación pasiva, ya que, según lo descrito por McNeil, las mismas compresiones serían efectivas para proveer ventilación al pacien- 


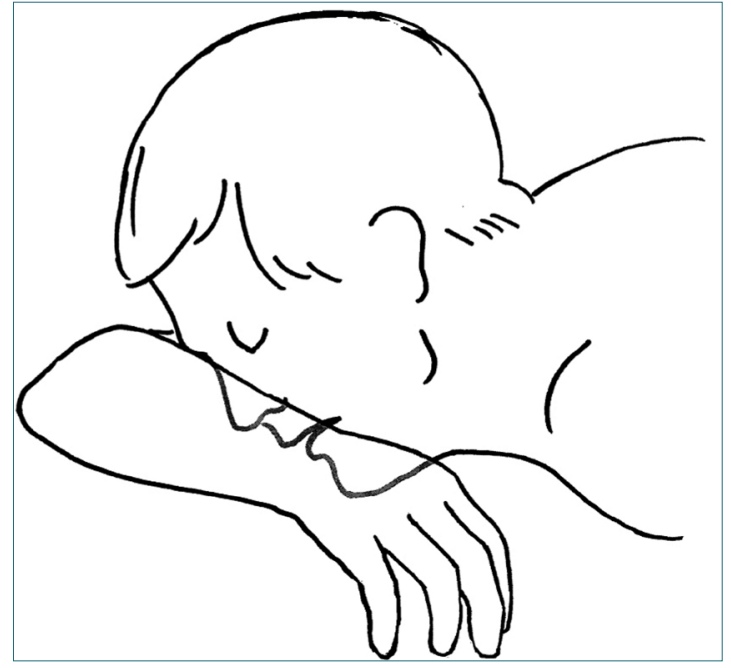

Figura 3. Posición del paciente en prono con la nariz hacia abajo.

te. Para este fin, se reclutaron 10 voluntarios sanos (5 médicos y 5 enfermeras) y se realizó una medición del volumen corriente exhalado mediante espirometría al realizarles compresiones sobre la espalda en posición prona, con la nariz pinzada y aguantando la respiración espontánea[9]. Pese a que este no era el escenario ideal para simular las condiciones de la reanimación durante un paro cardiorrespiratorio, se encontró que en los voluntarios se lograba un volumen corriente exhalado durante las compresiones que en promedio alcanzaba los $399 \pm 110 \mathrm{ml}[9]$. Si bien las condiciones en las que se realizó el estudio no permitirían homologar los valores cuantitativos observados, sí podría apoyar la teoría de que efectivamente existe una ventilación pasiva al realizar la técnica de RCP en prono.

\section{Desfibrilación en prono}

Respecto a la desfibrilación en el paciente en prono existen casos descritos donde se ha desfibrilado con éxito, con posterior retorno espontáneo de la circulación, a pacientes en posición prona sin haberlos cambiado previamente a posición supina. Miranda et al. publicaron, en el año 2001, el reporte del caso de una paciente que presentó fibrilación ventricular durante una cirugía de columna. Se describe que la paciente fue desfibrilada en posición prona con éxito utilizando dos parches estándar (3M) posicionados en la espalda de la paciente, uno en la región izquierda baja justo posterior a la línea axilar media y el otro justo bajo la escápula derecha, requiriendo solo una descarga de $200 \mathrm{~J}$ y posteriormente, volviendo a ritmo sinusal[11]. También se ha reportado éxito utilizando la posición antero-posterior para los parches o paletas, la cual fue descrita por Cattell et al., donde se posicionaba uno de los parches bajo el paciente, que se encuentra en prono, en relación a la zona de mayor masa ventricular y el otro en la espalda de este, específicamente sobre la escápula derecha[12],[13].

Debe considerarse de igual forma que el posicionamiento de parches en la espalda del paciente podría no entregar energía a una suficiente cantidad de miocardio durante la descarga debido al desplazamiento del corazón hacia adelante tanto por la posición prona como también por un posible aumento de la impedancia transtorácica con la ventilación a presión positiva[13],[14].

Se recomienda que si la desfibrilación fuese necesaria en pacientes que se encuentran siendo ventilados en posición prona esta debería intentarse primero en esta misma posición ya que el volver el paciente a supino consumiría minutos valiosos y reduciría la probabilidad de una desfibrilación exitosa[11],[15].

\section{Escenario actual: COVID-19}

La posición prona es una estrategia ampliamente utilizada en el manejo del SDRA severo en pacientes que se encuentran en ventilación mecánica invasiva[16]. En pacientes intubados con COVID-19 el prono temprano y prolongado (al menos $12 \mathrm{~h}$ diarias), mejora la oxigenación y disminuye la mortalidad[17]-[19]. Se ha visto que la característica principal de las infecciones severas por SARS-CoV-2 es el daño pulmonar agudo que se asemeja a un SDRA[20],[21], siendo esta la razón por la que la ventilación en posición prona actualmente se ha transformado en una estrategia frecuente tanto para pacientes despiertos como para aquellos en ventilación mecánica invasiva. Esta estrategia ha mostrado beneficio también en pacientes despiertos que mantienen ventilación espontánea siendo una importante estrategia para mejorar la oxigenación y prevenir un eventual traslado a UPC. Sin embargo, sus principales limitaciones serían que esta no es bien tolerada por períodos largos en pacientes despiertos y tampoco todos ellos obtienen beneficio de ella[22]. En la mayoría de los casos los pacientes que son ventilados en prono ya se encuentran intubados, sin embargo, dependiendo de la realidad local también puede existir un número no menor de pacientes despiertos con ventilación espontánea sien- 
do manejados en esta posición por lo que es importante contar siempre con un plan de acción ante un posible paro cardiorrespiratorio en estos pacientes.

Hasta el momento las principales sociedades internacionales han publicado guías de actualización haciendo referencia a la reanimación en pacientes COVID-19, como las del European Resuscitation Council y la guía provisoria de la American Heart Association (AHA)/Emergency Cardiovascular Care Committee (ECCC)[23],[24]. También han emanado sus recomendaciones el International Liaison Committee on Resuscitation (ILCOR) y el Resuscitation Council de Reino Unido (RCUK), las cuales, junto con las de la AHA son mencionadas en el artículo "Paro Cardiorrespiratorio y Reanimación Cardiopulmonar en un nuevo escenario: COVID-19" recientemente publicado en la Revista Chilena de Anestesia[25], por lo que, no han sido incluidas en esta revisión salvo aquellas de mayor relevancia para el contexto específico de pacientes en prono.

Respecto a la RCP en posición prona en pacientes COVID-19, dentro de sus posibles beneficios destaca que esta podría limitar la carga de trabajo y reducir los riesgos de exposición del personal durante la reanimación, ya sea derivados de los procedimientos propios de esta, la exposición de más personal a un ambiente altamente contaminado o por el riesgo de generación de aerosoles secundario a una posible desconexión o extubación accidental del paciente[26]. Pese a presentar beneficios claros, estos no necesariamente se traducirán en mejores resultados. Respecto al análisis de la evidencia existente, en julio del presente año fue publicado un artículo de revisión, realizado por Douma et al., sobre la RCP en prono, teniendo en cuenta el contexto de la actual pandemia[27]: en el artículo se concluye que hasta el momento no se ha encontrado suficiente evidencia como para dar lugar a una revisión sistemática o para reconsiderar las guías actuales, además señala que existen brechas en la evidencia en relación a la RCP en prono, principalmente una falta de evidencia de nivel moderado -alto, además de una escases de estudios sobre paro cardiorrespiratorio en contexto extrahospitalario[27].

Respecto a la reanimación en pacientes $\mathrm{CO}$ VID-19, específicamente aquellos que se encuentran siendo ventilados en posición prona, considerando la falta de evidencia actual, se han tomado las recomendaciones de los expertos publicadas en las diferentes guías. De acuerdo a estas recomendaciones se sugiere:

- Si el paciente se encuentra en prono sin una vía aérea avanzada, se recomienda intentar moverlo a posición supina antes de comenzar con la reani- mación[23],[24].

- En pacientes en prono que cuentan con una vía aérea avanzada, se recomienda evitar moverlo a posición supina a menos que esto pueda ser realizado sin riesgo de desconexión de equipos y aerosolización. Se debe proceder a colocar los parches del desfibrilador y realizar la RCP con el paciente en prono, colocando las manos en la posición habitual y comprimiendo sobre los cuerpos vertebrales de T7/T10[23] o entre las escápulas[24].

- Se sugiere supinar al paciente si:

- Las compresiones en prono no están siendo efectivas. Evaluar mediante la medición de la línea arterial (si está disponible) con un objetivo de presión diastólica > $25 \mathrm{mmHg}[24]$. La efectividad de las compresiones también puede ser evaluada con el $\mathrm{CO}_{2}$ al final de la espiración (etCO $\mathrm{C}_{2}$ )[27].

- Se requiere realizar intervenciones que requieren que el paciente esté en supino como, por ejemplo problemas con la vía aérea[24].

- Si no se logra un retorno de la circulación rápido (minutos)[24].

- Opciones de colocación de los parches del desfibrilador en el paciente en prono:

- Posición anterior-posterior[23],[24].

- Biaxilar (en ambas axilas del paciente)[24].

\section{Exposición y uso de elementos de protección personal (EPP)}

Actualmente, sabemos que el principal medio de transmisión del SARS-CoV-2 es mediante gotitas[28], sin embargo, también puede ocurrir la transmisión aérea principalmente cuando existe generación de aerosoles durante ciertos procedimientos como la intubación y la ventilación no invasiva[28],[29].

Es conocido que la reanimación añade aún más riesgo al personal de salud, principalmente debido a que la realización de la RCP involucra numerosos procedimientos generadores de aerosoles, incluyendo las compresiones torácicas, la ventilación a presión positiva y el establecimiento de una vía aérea avanzada[23]. Durante estos procedimientos, partículas virales pueden permanecer suspendidas en el aire con una vida media de aproximadamente $1 \mathrm{~h}$ y ser inhaladas por quienes se encuentren cerca[23],[30]. Otro factor de riesgo a tener en cuenta es que usualmente los esfuerzos de reanimación requieren de numeroso personal trabajando en proximidad entre sí y con el paciente. Estos eventos de emergencia en los que el paciente requiere medidas de reanimación inmediatas son altamente estresantes lo cual puede traducirse en 
fallas en el seguimiento de los protocolos de control de infecciones y de uso correcto de EPP[23]. Una posible solución a este problema sería el designar a un miembro del equipo el rol específico de "observador" y que estaría encargado de asegurar el correcto uso de las EPP por parte del resto de los miembros del equipo de reanimación[28].

El Australasian College for Emergency Medicine desarrolló guías para el manejo del paro cardiorrespiratorio en pacientes adultos COVID-19 para ser aplicadas a la población de Australia y Nueva Zelanda, en estas se incluye una guía simple y concisa sobre el uso de EPP para diversos procedimientos de acuerdo al riesgo documentado para la transmisión del virus en pacientes COVID-19[28]. Estas recomendaciones han sido adaptadas e incluidas a continuación:

- Las EPP para bajo riesgo de transmisión deben ser usadas para todos los procedimientos e incluye: Mascarilla quirúrgica (o N95 si se encuentra disponible), protección ocular y guantes.

- Las EPP de gotitas agregan el uso de delantal a las de bajo riesgo y se recomienda usar en los siguientes procedimientos:

- Primer respondedor (reconocimiento del paro cardiorrespiratorio y solicitud de ayuda).

- Colocación de mascarilla de oxígeno al paciente (con flujo hasta $10 \mathrm{~L} / \mathrm{min}$ ) cubierta con toalla, tela, sábana plástica o mascarilla quirúrgica.

- Desfibrilación (con la cara del paciente cubierta).

- RCP inicial por parte del primer respondedor (sólo compresiones) mientras espera la llegada de personal con EPP aéreas.

- Maniobras básicas de vía aérea (levantamiento del mentón, inclinación de la cabeza, luxación de la mandíbula).

- Las EPP de aerosol/aéreas incluyen todas las de gotitas y agregan el uso obligatorio de mascarilla N95, escudo facial/visor, gorro y protección del cuello, pudiendo ajustarse según las guías locales. Se recomiendan para los siguientes procedimientos:

- Continuación de las compresiones torácicas durante la RCP (iniciadas por el primer respondedor).

- Colocación de vía aérea orofaríngea o nasofaríngea.

- Ventilación con bolsa - mascarilla.

- Colocación de vía aérea supraglótica.

- Intubación.

Se recomienda utilizar mascarilla N95 siempre que se encuentre disponible. Aquellos pacientes en los que no se pueda determinar el riesgo de transmisión derivado del procedimiento debiesen considerarse como de alto riesgo.

La guía del Australasian College for Emergency Medicine señala que estas recomendaciones son realizadas con un enfoque conservador ya que actualmente, existe poca evidencia sobre la capacidad de generación de aerosoles de cada procedimiento realizado durante la reanimación, por lo que es actualmente motivo de debate[28].

\section{Discusión}

Las técnicas de RCP clásica es uno de los conocimientos en medicina que no ha experimentado grandes ni numerosos cambios con el pasar de los años, pese a que muchas veces se ha puesto en duda su efectividad. Ha habido intentos de generar cambios en esta misma, sin embargo, ninguna técnica nueva ha generado un impacto suficiente para motivar un cambio. Si bien a la fecha no existe mucha experiencia ni evidencia respecto a las técnicas y a la efectividad de la RCP en prono, los fundamentos científicos en los que se basan parecen razonables y los beneficios que, según se planteó, presentarían estas mismas por sobre las clásicas, las vuelve una opción viable para investigar en pos del desarrollo de nuevas técnicas. De igual manera, la situación actual obliga a tener en cuenta los conocimientos con los que se cuenta hasta la fecha como una opción o alternativa ante la necesidad de realizar una reanimación en prono, lo cual actualmente no es solo una real posibilidad, sino que un escenario que a muchos les tocará vivir. Respecto a lo expuesto, si tenemos en cuenta el nivel anatómico ideal para la compresión en prono descrito previamente, junto con la técnica desarrollada originalmente por McNeil y los beneficios que teóricamente esta proporcionaría, la volverían una opción factible de aplicar en el tiempo actual sin requerir mayor entrenamiento adicional y siendo fácil de aplicar incluso por un solo reanimador. Por estas razones es que parecería factible considerar como alternativa la realización de una técnica de compresión similar, teniendo en cuenta, además, la localización ideal de las manos para lograr una mayor compresión del ventrículo izquierdo, en busca de lograr una técnica de RCP en prono que maximice los beneficios y disminuya los riesgos para el paciente, sin generar riesgos asociados a su aplicación. Si bien no se cuenta con evidencia suficiente para avalar la superioridad de la técnica en prono, en la situación que vivimos en este momento muchas veces realizar la reanimación en prono será la única opción, lo que resulta muchas veces muy complejo, pero también nos abre una puerta a la oportu- 
nidad de aprender, evaluar y registrar la efectividad de las técnicas de RCP en prono.

Respecto al paciente COVID-19 específicamente, podría resultar poco efectivo el realizar la RCP en prono sin un dispositivo de vía aérea que permita asegurar su adecuada ventilación ya que en muchos casos la misma causa del paro cardiorrespiratorio será la hipoxia, razón por la cual es imprescindible asegurar una óptima ventilación durante la reanimación. El otro factor importante a considerar es el riesgo de contagio y el tiempo que toma el colocarse los elementos de protección personal lo cual muchas veces obliga a poner en la balanza el riesgo personal y el riesgo para el paciente derivado de una demora en el inicio de la RCP. Por lo antes expuesto es que es de suma importancia contar con un plan de acción local habiendo discutido previamente con el equipo de trabajo tanto los riesgos como las acciones que deben realizarse expeditamente, evitando así el tener que decidir esto en el momento en que el tiempo es más crucial.

Para finalizar, me gustaría invitar a todos, sobretodo a nuestros especialistas, a compartir sus diferentes experiencias con el objetivo de poder ampliar la evidencia existente sobre este tema y de esta manera aprovechar al máximo las oportunidades que nos brinda esta pandemia, aumentando de esta forma los conocimientos con los que contamos hasta el momento, promoviendo el desarrollo de nuevas directrices y motivando los cambios que sean necesarios para de esta forma lograr a futuro una RCP de la mayor calidad y efectividad posible.

\section{Referencias}

1. Sun $W Z$, Huang $F Y$, Kung $K L$, Fan SZ, Chen TL. Successful cardiopulmonary resuscitation of two patients in the prone position using reversed precordial compression. Anesthesiology. 1992 Jul;77(1):202-4. https:// doi.org/10.1097/00000542199207000-00027 PMID:1609994

2. Tobias JD, Mencio GA, Atwood R, Gurwitz GS. Intraoperative cardiopulmonary resuscitation in the prone position. J Pediatr Surg. 1994 Dec;29(12):1537-8. https://doi.org/10.1016/00223468(94)90208-9 PMID:7877020

3. Dequin PF, Hazouard E, Legras A, Lanotte R, Perrotin D. Cardiopulmonary resuscitation in the prone position: kouwenhoven revisited. Intensive Care Med. 1996 Nov;22(11):1272. https:// doi.org/10.1007/BF01709349 PMID:9120126

4. McNeil EL. Re-evaluation of cardiopulmonary resuscitation. Resuscitation. 1989 Oct;18(1):15. https://doi.org/10.1016/03009572(89)90107-X

\section{PMID:2554441}

5. McNeil EL. Re-evaluation of cardiopulmonary resuscitation. Resuscitation. 2003 Feb;56(2):22930. https://doi.org/10.1016/ S0300-9572(02)00406-9 PMID:12589999

6. Stewart JA. Resuscitating an idea: prone CPR. Resuscitation. 2002 Sep;54(3):231-6. https://doi.org/10.1016/ s0300-9572(02)00145-4 PMID:12204455

7. Redberg RF, Tucker KJ, Cohen TJ, Dutton JP, Callaham ML, Schiller NB. Physiology of blood flow during cardiopulmonary resuscitation. A transesophageal echocardiographic study. Circulation. 1993 Aug;88(2):534-42. https://doi. org/10.1161/01.CIR.88.2.534 PMID:8339415

8. Kwon MJ, Kim EH, Song IK, Lee JH, Kim HS, Kim JT. Optimizing Prone Cardiopulmonary Resuscitation: Identifying the Vertebral Level Correlating With the Largest Left Ventricle CrossSectional Area via Computed Tomography Scan. Anesth Analg. 2017 Feb;124(2):5203. https://doi.org/10.1213/

\section{ANE.0000000000001369} PMID:27454066

9. Wei J, Tung D, Sue SH, Wu SV, Chuang YC, Chang CY. Cardiopulmonary resuscitation in prone position: a simplified method for outpatients. J Chin Med Assoc. 2006 May;69(5):2026. https://doi.org/10.1016/ S1726-4901(09)70219-9 PMID:16835981

10. Safar P, Escarraga LA, Chang F. Upper airway obstruction in the unconscious patient. J Appl Physiol. 1959 Sep;14(5):7604. https://doi.org/10.1152/ jappl.1959.14.5.760 PMID:14440737

11. Miranda CC, Newton MC. Successful defibrillation in the prone position. Br J Anaesth. 2001 Dec;87(6):937-8. https:// doi.org/10.1093/bja/87.6.937 PMID:11878701

12. Cattell E, Saravanan P, Chay S, Lawler PG. The defibrillator back paddle: use for treatment of arrhythmias during prone position ventilation. Anaesthesia. 2000 May;55(5):491. https:// doi.org/10.1046/j.13652044.2000.01425-6.x PMID:10792918 
13. Edgcombe $\mathrm{H}$, Carter K, Yarrow $\mathrm{S}$. Anaesthesia in the prone position. Br J Anaesth. 2008 Feb;100(2):165-83. https:// doi.org/10.1093/bja/aem380 PMID:18211991

14. Walsh SJ, Bedi A, Miranda C. Successful defibrillation in the prone position. Br J Anaesth. 2002 Nov;89(5):799-800. https:// doi.org/10.1093/bja/89.5.799 PMID:12393790

15. Bossaert L, Callanan V, Cummins RO. Early defibrillation. An advisory statement by the Advanced Life Support Working Group of the International Liaison Committee on Resuscitation. Resuscitation. 1997 Apr;34(2):1134. https://doi.org/10.1016/ S0300-9572(97)01101-5 PMID:9141156

16. Bloomfield R, Noble DW, Sudlow A. Prone position for acute respiratory failure in adults. Cochrane Database Syst Rev. 2015 Nov;(11):CD008095. https:// doi.org/10.1002/14651858. CD008095.pub2 PMID:26561745

17. Elharrar $X$, Trigui $Y$, Dols AM, Touchon F, Martinez $S$, Prud'homme E, et al. Use of Prone Positioning in Nonintubated Patients With COVID-19 and Hypoxemic Acute Respiratory Failure. JAMA. 2020 Jun;323(22):2336-8. https://doi. org/10.1001/jama.2020.8255; Epub ahead of print. PMID:32412581

18. Munshi L, Del Sorbo L, Adhikari NK, Hodgson CL, Wunsch $\mathrm{H}$, Meade $\mathrm{MO}$, et al. Prone Position for Acute Respiratory Distress Syndrome. A Systematic Review and Meta-Analysis. Ann Am Thorac Soc. 2017 Oct;14 Supplement_4:\$280-8. https://doi.org/10.1513/ AnnalsATS.201704-3430T PMID:29068269

19. Guérin C, Reignier J, Richard
$J C$, Beuret P, Gacouin A, Boulain $T$, et al.; PROSEVA Study Group. Prone positioning in severe acute respiratory distress syndrome. N Engl J Med. 2013 Jun;368(23):2159-68. https://doi. org/10.1056/NEJMoa1214103 PMID:23688302

20. Barker J, Koeckerling D, West R. A need for prone position CPR guidance for intubated and non-intubated patients during the COVID-19 pandemic. Resuscitation. 2020 Jun;151:1356. https://doi.org/10.1016/j. resuscitation.2020.04.029 PMID:32371028

21. Gattinoni L, Coppola S, Cressoni M, Busana M, Rossi S, Chiumello D. COVID-19 Does Not Lead to a "Typical" Acute Respiratory Distress Syndrome. Am J Respir Crit Care Med. 2020 May;201(10):1299-300. https:// doi.org/10.1164/rccm.2020030817LE PMID:32228035

22. Elharrar $X$, Trigui $Y$, Dols AM, Touchon F, Martinez $S$, Prud'homme E, et al. Use of Prone Positioning in Nonintubated Patients With COVID-19 and Hypoxemic Acute Respiratory Failure. JAMA. 2020 Jun;323(22):2336-8. https://doi. org/10.1001/jama.2020.8255; Epub ahead of print. PMID:32412581

23. Edelson DP, Sasson C, Chan PS, Atkins DL, Aziz K, Becker LB, et al.; American Heart Association ECC Interim COVID Guidance Authors. Interim Guidance for Basic and Advanced Life Support in Adults, Children, and Neonates With Suspected or Confirmed COVID-19: From the Emergency Cardiovascular Care Committee and Get With The GuidelinesResuscitation Adult and Pediatric Task Forces of the American Heart Association. Circulation. 2020 Jun;141(25):e933-43. https://doi.org/10.1161/CIR-
CULATIONAHA.120.047463

PMID:32270695

24. Nolan JP, Monsieurs KG, Bossaert L, Böttiger BW, Greif R, Lott $C$, et al.; European Resuscitation Council COVID-Guideline Writing Groups. European Resuscitation Council COVID-19 guidelines executive summary. Resuscitation. 2020 Aug;153:4555. https://doi.org/10.1016/j. resuscitation.2020.06.001 PMID:32525022

25. Aguirre M. Paro Cardiorrespiratorio (PCR) y Reanimación Cardiopulmonar (RCP) en un nuevo escenario: COVID19. Revista Chilena de Anestesia. 2020;49(3):388-96. https:// doi.org/10.25237/revchilanestv49n03.017.

26. Medrzycka-Dabrowska W, Lewandowska K, Dlezak D, Dabrowski S. Prone ventilation of critically ill adults with $\mathrm{CO}$ VID-19: how to perform CPR in cardiac arrest? Crit Care. 2020 May;24(1):258. https:// doi.org/10.1186/s13054-02002970-y PMID:32456664

27. Douma MJ, MacKenzie E, Loch T, Tan MC, Anderson D, Picard $C$, et al. Prone cardiopulmonary resuscitation: A scoping and expanded grey literature review for the COVID-19 pandemic. Resuscitation. 2020 Oct;155:10311. https://doi.org/10.1016/j. resuscitation.2020.07.010 PMID:32707142

28. Craig $S$, Cubitt $M$, Jaison $A$, Troupakis S, Hood N, Fong C, et al. Management of adult cardiac arrest in the COVID-19 era: consensus statement from the Australasian College for Emergency Medicine. Med J Aust. 2020 Aug;213(3):12633. https://doi.org/10.5694/ mja2.50699; Epub ahead of print. PMID:32656798

29. World Health Organization. (2020). Rational use of personal 
protective equipment for coronavirus disease (COVID-19): interim guidance, 27 February 2020.

World Health Organization.

https://apps.who.int/iris/hand- le/10665/331215.

30. van Doremalen N, Bushmaker T, Morris DH, Holbrook MG, Gamble A, Williamson BN, et al. Aerosol and Surface Stability of SARS-CoV-2 as Compared with SARS-CoV-1. N Engl J Med. 2020 Apr;382(16):1564-7. https://doi.org/10.1056/NEJMc2004973 PMID:32182409 